\title{
Comparative Toxicities of \\ Diphtherial Toxin and Pseudomonas aeruginosa Exotoxin A: Evidence for Different Cell Receptors
}

\author{
By MICHAEL L. VASIL* AND BARBARA H. IGLEWSKI \\ Department of Microbiology and Immunology, University of Oregon \\ Health Sciences Center, Portland, Oregon 97201, U.S.A.
}

(Received 28 March 1978; revised 31 May 1978)

\section{INTRODUCTION}

Exotoxin A of Pseudomonas aeruginosa (PA toxin) inhibits intracellular protein synthesis in mammalian cells by a very similar if not identical mechanism to diphtherial toxin (Iglewski et al., 1976; Iglewski \& Kabat, 1975; Iglewski et al., 1977). This inhibition of protein synthesis requires NAD and results in the inactivation of elongation factor 2 (EF-2). Specifically, PA toxin or its fragment A (Vasil et al., 1977; Chung \& Collier, 1977) like the fragment A of diphtherial toxin (Collier, 1975) catalyses the transfer of the adenosine diphosphate ribose moiety (ADP-ribose) of NAD on to EF-2. The resultant ADP-riboseEF-2 complex is inactive in protein synthesis. There are also similarities between PA toxin and diphtherial toxin with regard to structure-function relationships (Vasil et al., 1977; Chung \& Collier, 1977). PA toxin (mol. wt 71 500) and diphtherial toxin (mol. wt 63000) are both single polypeptides and are found in culture supernatants in a toxic but nonenzymically active form (proenzyme). Each toxin consists of an enzymically active component (ADP-ribosyl transferase) necessary for intracellular inhibition of protein synthesis and a carrier component termed fragment $B$ (binding) which is thought to be responsible for making initial contact with a cell surface receptor. It should be noted that PA toxin can be separated into A and B fragments, but it is not clear whether this type of fragmentation is actually required for expression of toxicity of PA toxin as it appears to be for diphtherial toxin (Vasil et al., 1977; Leppla, 1976).

The A fragments of PA toxin and diphtherial toxin have been isolated and characterized (Collier, 1975) but the relative insolubility of the $\mathrm{B}$ fragments in the absence of dispersing agents has limited the study of these components (Collier, 1975; Vasil et al., 1977). It was recently reported (Zanen et al., 1976) that the B fragment of diphtherial toxin remains soluble after extensive dialysis against a $0.02 \mathrm{M}$-borate buffer. The fragment B of diphtherial toxin so obtained can compete with the intact toxin for the specific receptors on susceptible cells (Zanen et al., 1976; Everse et al., 1977). If the B fragment is added in sufficient quantities it can reduce the cytotoxicity and/or the inhibition of cellular protein synthesis caused by diphtherial toxin (Everse et al., 1977; Zanen et al., 1976). Similar competition studies have also been done using a non-toxic protein (CRM 197) which cross-reacts with diphtherial toxin and contains an inactive fragment $A$ and a functional fragment $B$ (Uchida et al., 1973). Thus far, no one has successfully isolated the B fragment of PA toxin, and non-toxic CRM proteins (similar to CRM 197) are not yet available.

While the available literature pertaining to the action of diphtherial toxin on intact cells and experimental animals is extensive (see review by Solotorovsky \& Johnson, 1970), there is only a limited amount of information regarding the cellular and animal specificities of

* Present address: Department of Microbiology and Immunology, University of Colorado School of Medicine, Denver, Colorado 80262, U.S.A. 
PA toxin (Middlebrook \& Dorland, 1977a, b; Pavlovskis et al., 1974; Pavlovskis \& Gordon, 1972) and direct comparative studies between PA toxin and diphtherial toxin are few (Middlebrook \& Dorland, 1977a). These studies did not directly measure inhibition of protein synthesis and kinetic studies have not been done with a cell line that is equally sensitive to both toxins.

In the present study, the comparative toxicities of PA toxin and diphtherial toxin were examined by measuring inhibition of protein synthesis in cultured cells. The kinetics of inhibition of protein synthesis by these toxins were compared in a cell line that is apparently equally susceptible to both toxins. In addition, experiments were done to determine if the diphtherial CRM 197 protein could compete with PA toxin thereby inhibiting the action of PA toxin on protein synthesis in cell culture.

\section{METHODS}

Preparation of PA toxin, diphtherial toxin and CRM 197 protein. PA toxin and diphtherial toxin were produced and purified in this laboratory as previously described (Iglewski et al., 1976; Leppla, 1976; Vasil et al., 1977). The PA toxin appeared as a single protein band when a $25 \mu \mathrm{g}$ sample was analysed by electrophoresis on polyacrylamide gels containing sodium dodecyl sulphate (Vasil et al., 1977). This toxin had a mouse mean lethal dose $\left(\mathrm{LD}_{50}\right)$ of $0.2 \mu \mathrm{g}$ when injected intraperitoneally into $22 \mathrm{~g}$ female Swiss-Webster mice. The purified diphtherial toxin contained 15 guinea-pig lethal doses per $\mu$ g protein. CRM 197 protein was a kind gift of T. Uchida (Osaka University, Osaka, Japan). The CRM 197 preparation showed a single protein band of molecular weight 63000 (Collier, 1975) when a $25 \mu \mathrm{g}$ sample was analysed by electrophoresis on polyacrylamide gels containing sodium dodecyl sulphate (unpublished observations). The immunological identity of CRM 197 with diphtherial toxin was confirmed by Ouchterlony immunodiffusion analysis.

Inhibition of protein synthesis by PA toxin and diphtherial toxin in cell culture. HeLa cells, Hep-2 cells, Chang liver (human) cells and mouse L-929 cells were obtained from the American Type Culture Collection. Cells were grown in Eagle's minimum essential medium containing $10 \%(\mathrm{v} / \mathrm{v})$ foetal calf serum and $1 \%$ (w/v) glutamine. Primary cultures of chick embryo fibroblasts were prepared from 9 to $11 \mathrm{~d}$-old c/o type embryos as previously described (Iglewski et al., 1975). The chick cells were seeded in medium 199 supplemented with tryptose phosphate broth $(19 \%)$, foetal calf serum $(8 \%)$ and heated $\left(56{ }^{\circ} \mathrm{C}, 30\right.$ min) chicken serum $(2 \%)$. Secondary cultures were prepared by treating primary cultures with trypsin and seeded at $10^{6}$ cells per $35 \mathrm{~mm}$ Petri dish for the assay of inhibition of protein synthesis.

Inhibition of protein synthesis on the various cell lines was assayed as previously described (Vasil et al., 1976). The $\mathrm{TCLD}_{50}$ values and the standard error of means presented in Table 1 are based on three or four independent assays which were each done in triplicate. ${ }^{3} \mathrm{H}$-Labelled amino acids were obtained from New England Nuclear (NET-250) and used as previously described (Vasil et al., 1976).

\section{RESULTS AND DISCUSSION}

The sensitivities of chicken, mouse and human cell lines to PA toxin and diphtherial toxin varied independently with respect to the type of toxin (Table 1). Thus, some cell lines were more sensitive to PA toxin and some cell lines were equally sensitive to both toxins. For example, HeLa cells were about 30 -fold and Hep-2 cells were about eightfold more sensitive to diphtherial toxin than they were to PA toxin. A similar relationship between diphtherial toxin and PA toxin has also been seen with baby hamster kidney (BHK) cells (W. J. Iglewski \& A. K. Taylor, personal communications). In contrast, the mouse L-cells were more than 500 times more sensitive to PA toxin than they were to diphtherial toxin. Secondary cultures of chick embryo fibroblasts were equally sensitive to PA toxin or diphtherial toxin by this assay.

Another interesting relationship is seen with the human cell lines. The HeLa and Hep-2 cells were more sensitive to diphtherial toxin than to PA toxin, while the reverse was true for the Chang liver cells. Thus, of the three human cell lines examined, the Chang liver cells were the most sensitive to PA toxin. It is possible that the liver may be a 'target organ' in experiments carried out on mice and perhaps this might be the case also in some human infections (Iglewski et al., 1977; Pavlovskis et al., 1978). 
Table 1. Comparative toxicities of PA toxin and diphtherial toxin on cell cultures

Cell monolayers were treated with various concentrations $\left(0.002\right.$ to $\left.20.0 \mu \mathrm{g} \mathrm{ml}^{-1}\right)$ of PA toxin or diphtherial toxin for $4 \mathrm{~h}$, then pulsed for $1 \mathrm{~h}$ with ${ }^{3} \mathrm{H}$-labelled amino acids.

Cell line

HeLa

Mouse L-929

Hep-2 (human)

Chang liver (human)

Chick embryo (secondary culture)
TCLD $_{50} * \pm$ standard error of the mean

$\begin{array}{lc}\text { PA toxin } & \text { Diphtherial toxin } \\ 0.53 \pm 0.1 & 0.015 \pm 0.0006 \\ 0.036 \pm 0.016 & >20.0 \\ 0.33 \pm 0.06 & 0.045 \pm 0.01 \\ 0.058 \pm 0.01 & 0.8 \pm 0.12 \\ 0.02 \pm 0.009 & 0.02 \pm 0.008\end{array}$

* Tissue culture mean lethal dose, i.e. the amount of toxin $\left(\mu \mathrm{g} \mathrm{ml}^{-1}\right)$ required to inhibit protein synthesis by $50 \%$ in about $1 \times 10^{6}$ cells.
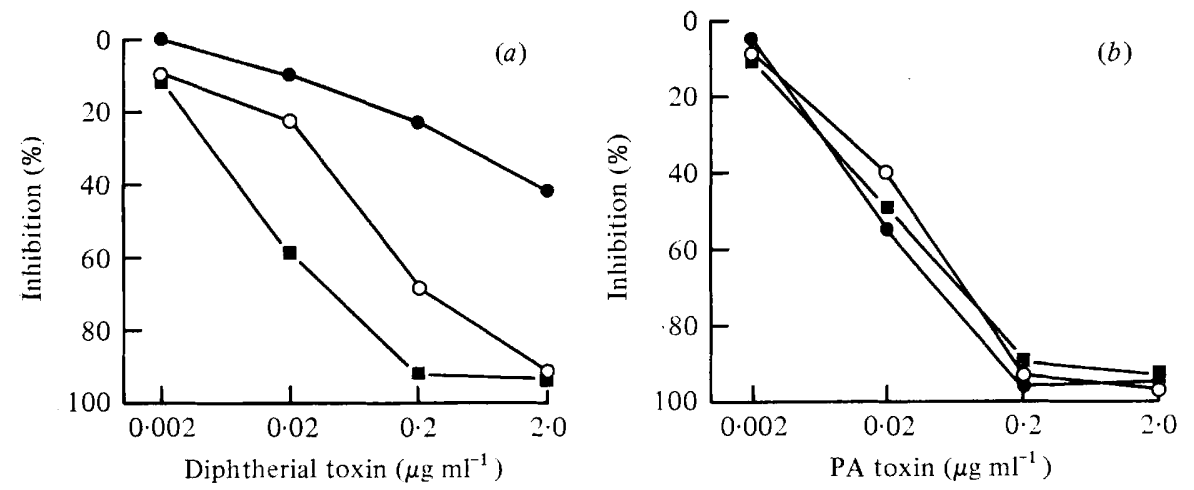

Fig. 1. Effect of CRM 197 protein on the inhibition of protein synthesis in secondary chick embryo fibroblasts by diphtherial toxin $(a)$ and by PA toxin $(b)$. CRM 197 at $0 \mu \mathrm{g} \mathrm{ml}^{-1}$ (घ), $1.0 \mu \mathrm{g} \mathrm{ml}^{-1}(\mathrm{O})$, or $2.5 \mu \mathrm{g} \mathrm{ml}^{-1}(\mathrm{O})$ was added to monolayers of cells for $5 \mathrm{~min}$ prior to treatment of cells with various concentrations of diphtherial toxin or PA toxin. The inhibition of protein synthesis was determined by measuring the percentage reduction of ${ }^{3} \mathrm{H}$-labelled amino acid incorporation compared with incorporation in controls to which no toxin or only CRM 197 was added. There was no significant difference in the amount of incorporation of ${ }^{3} \mathrm{H}$-labelled amino acids, as measured by radioactivity (c.p.m.), into these two kinds of control cells, even with the highest concentration of CRM 197 tested $\left(5 \mu \mathrm{g} \mathrm{ml}^{-1}\right)$.

Even though the chick embryo fibroblasts were equally sensitive to diphtherial toxin and PA toxin by the assay that was used, it was still possible that the rates of intoxication by the toxins might differ due to transport and/or internalization of the A portions of the toxins. However, there appeared to be no significant difference in the rate of inhibition of protein synthesis between cells treated with diphtherial toxin and those treated with PA toxin (data not shown). Thus, this cell line was chosen for further studies on the mechanism of intoxication by PA toxin and diphtherial toxin.

The fact that different cells had different sensitivities to PA toxin and diphtherial toxin suggests that there are different cell surface receptors for each toxin. Other mechanisms such as processing and/or transport could also account for these differences but these processes are complex and difficult to examine. With the availability of CRM 197 which cross-reacts immunologically with diphtherial toxin and which is known to compete with diphtherial toxin for binding to eukaryotic cells (Collier, 1975; Uchida et al., 1973), it was possible to examine whether PA toxin utilizes the same receptors as diphtherial toxin.

Figure 1 $(a)$ presents the data from the control experiment demonstrating that the CRM 197 preparation was capable of inhibiting the action of diphtherial toxin on the protein synthesis of chick embryo fibroblasts. As the concentration of CRM 197 was 
increased, the effect of diphtherial toxin on protein synthesis was dramatically decreased. Figure $1(b)$, in contrast, shows that even at the highest concentration of CRM $197(2.5 \mu \mathrm{g})$ the amount of PA toxin required to inhibit protein synthesis in chick embryo fibroblasts remained unchanged. Concentrations of CRM 197 as high as $5.0 \mu \mathrm{g}$ also showed no effect (unpublished observations). Thus, CRM 197 did not compete with PA toxin in this system indicating that the receptors for diphtherial toxin and PA toxin are different. Similar results have been obtained using a partially purified preparation of the fragment $B$ of diphtherial toxin (M. Vasil, unpublished observations). Because the fragment B of PA toxin has only been tentatively identified (Vasil et al., 1977) and a non-toxic immunologically identical CRM of PA toxin is not yet available, the reverse experiment cannot yet be done.

It is evident that there are some significant differences between the toxicities of PA toxin and diphtherial toxin even though they have very similar if not identical enzymic activities (Iglewski et al., 1976; Iglewski \& Kabat, 1975; Iglewski et al., 1977) and several similarities in structure-function relationships (Vasil et al., 1977; Chung \& Collier, 1977). These differences between PA toxin and diphtherial toxin may involve the following: (i) different cell membrane receptors for each toxin; (ii) different mechanisms involved in activation of the proenzyme forms; or (iii) different mechanisms involved in transport or internalization of the enzymically active component. One or all of these different mechanisms may be operative and none is mutually exclusive of the others.

Because the A fragments of PA toxin and diphtherial toxin appear to function identically, it is likely that the distinct activities of these toxins on intact cells and animals is related to differences in the B components of these toxins and to differences in the receptors for the toxins.

That there are differences in the B components is supported by the failure to detect any immunological cross-reactivity between PA toxin and diphtherial toxin (Iglewski et al., 1976; Iglewski \& Kabat, 1975; Leppla, 1976). But immunological differences in the B components are not sufficient to rule out the possibility that these toxins use the same or a similar receptor. Thus, the plant lectins, concanavalin $\mathrm{A}$ and wheat germ agglutinin have recently been shown to competitively inhibit the action of diphtheria toxin on Chinese hamster V79 cells, yet no immunological cross-reactivity is known to exist between these molecules (Draper et al., 1978). The data from the CRM 197 experiments offer the most direct evidence that PA toxin and diphtherial toxin interact with different membrane receptors on susceptible cells. However, it is not yet clear if additional mechanisms such as transport of these toxins across the cell membrane are also involved in the differences between the expression of toxicity of PA toxin and diphtherial toxin. With the availability of a cell line that is equally sensitive to both toxins (e.g. secondary chick embryo fibroblasts), it may be possible to examine these basic mechanisms.

This study was supported by a grant from the National Cystic Fibrosis Society. M. L.V. was the recipient of an N.L. Tartar Research Fellowship Award from the Medical Research Foundation of Oregon and a Public Health Service Postdoctoral Fellowship (IF32 AI 9539701) of the National Institute of Allergy and Infectious Diseases; B.H.I. was a Senior National Research Foundation Fellow. We gratefully acknowledge the excellent technical assistance of Jerry Tolle and Sofia Chung Fagan.

\section{REFERENCES}

Chung, D. W. \& Collier, R. J. (1977). Enzymatically active peptide from the adenosine diphosphate-ribosylating toxin of Pseudomonas aeruginosa. Infection and Immunity 16, 832-841.

Collier, R. J. (1975). Diphtheria toxin: mode of action and structure. Bacteriological Reviews 39, 54-85.

Draper, R. K., Chen, D. \& Simon, M. I. (1978). Diphtheria toxin has the properties of a lectin.
Proceedings of the National Academy of Sciences of the United States of America 75, 261-265.

Everse, J., LaPPi, D. A., BeglaU, J. M., Lee, C. L. \& KAPLAN, N. O. (1977). Investigations into the relationship between structure and function of diphtheria toxin. Proceedings of the National Academy of Sciences of the United States of America 74, 472-476.

IGLEwSKI, B. H. \& KABAT, D. (1975). NAD-de- 
pendent inhibition of protein synthesis by Pseudomonas aeruginosa toxin. Proceedings of the National Academy of Sciences of the United States of America 72, 2284-2288.

Igiewski, B. H., RitTenberg, M. B. \& IGlewski, W. J. (1975). Preferential inhibition of growth and protein synthesis in Rous Sarcoma Virus transformed cells by diphtheria toxin. Virology $\mathbf{6 5}$, 272-275.

IGlewsKi, B. H., Elwell, L. P., Lui, P. V. \& KABAT, D. (1976). ADP-ribosylation of elongation factor 2 by Pseudomonas aeruginosa exotoxin A and by diphtheria toxin. In Proceedings of the 4th International Symposium on the Metabolic Interconversion of Enzymes, pp. 150-155. Edited by $\mathrm{S}$. Shaltiel. Berlin \& New York: SpringerVerlag.

Iglewski, B. H., Lui, P. V. \& Kabat, D. (1977). Mechanism of action of Pseudomonas aeruginosa exotoxin A adenosine diphosphate-ribosylation of mammalian elongation factor-2. Infection and Immunity 15, 138-144.

LEPPLA, S. H. (1976). Large-scale purification and characterization of the exotoxin of Pseudomonas aeruginosa. Infection and Immunity 14, 1077-1086.

MiddlebrooK, J. L. \& Dorland, R. B. (1977a). Response of cultured mammalian cells to the exotoxins of Pseudomonas aeruginosa and Corynebacterium diphtheriae: differential cytotoxicity. Canadian Journal of Microbiology 23, 183-189.

MiddlebrooK, J. L. \& Dorland, R. B. (1977b). Differential chemical protection of mammalian cells from the exotoxins of Corynebacterium diphtheriae and Pseudomonas aeruginosa. Infection and Immunity 16, 232-239.
Pavlovskis, O. R. \& Gordon, F. B. (1972). Pseudomonas aeruginosa: effect on cell cultures. Journal of Infectious Diseases 125, 631-636.

Pavlovskis, O. R., Callahan, L. T. \& Meyer, R. D. (1974). Characterization of exotoxin of Pseudomonas aeruginosa. Journal of Infectious Diseases 130 (suppl.) S100-S102.

Pavlovskis, O. R., Iglewski, B. H. \& Pollack, M. (1978). Mechanism of action of Pseudomonas aeruginosa exotoxin $\mathrm{A}$ in experimental mouse infections: adenosine diphosphate ribosylation of elongation factor 2 . Infection and Immunity 19 , 29-33.

Solotorovsky, M. \& Johnson, W. (1970). Tissue culture and bacterial protein toxins. In Microbial Toxins, vol. 1, pp. 277-327. Edited by S. J. Ajl, S. Kadis \& T. C. Montie. New York: Academic Press.

UChida, T., Pappenheimer, A. M., Jr \& Harper, A. A. (1973). Diphtheria toxin and related proteins. II. Kinetic studies on intoxication of HeLa cells by diphtheria toxin and related proteins. Journal of Biological Chemistry 248, 3845-3850.

VAsIL, M. L., LUI, P. V. \& IGLewSKI, B. H. (1976). Temperature-dependent inactivating factor of Pseudomonas aeruginosa exotoxin A. Infection and Immunity 13, 1467-1472.

Vasil, M. L., Kabat, D. \& Iglewski, B. H. (1977). Structure activity relationships of an exotoxin of Pseudomonas aeruginosa. Infection and Immunity 16, 353-361.

Zanen, J., MuYldermans, G. \& Beugnier, N. (1976). Competitive antagonists of the action of diphtheria toxin in HeLa cells. FEBS Letters 66, 261-263. 